\title{
Glasslike dynamical behavior in hierarchical models submitted to continuous cooling and heating processes
}

\author{
A. Prados and J. J. Brey \\ Física Teórica, Facultad de Física, Universidad de Sevilla, Apartado de Correos 1065, E-41080 Sevilla, Spain
}

(Received 15 March 2001; published 24 September 2001)

\begin{abstract}
The dynamical behavior of a kind of models with hierarchically constrained dynamics is investigated. The models exhibit many properties resembling real structural glasses. In particular, we focus on the study of time-dependent temperature processes. In cooling processes, a phenomenon analogous to the laboratory glass transition appears. The residual properties are analytically evaluated, and the concept of fictive temperature is discussed on a physical basis. The evolution of the system in heating processes is governed by the existence of a normal solution of the evolution equations, which is approached by all the other solutions. This trend of the system is directly related to the glassy hysteresis effects shown by these systems. The existence of the normal solution is not restricted to the linear regime around equilibrium, but it is defined for any arbitrary, far-fromequilibrium, situation.
\end{abstract}

DOI: 10.1103/PhysRevE.64.041505

PACS number(s): 64.70.Pf, 05.70.Ln, 45.70.-n

\section{INTRODUCTION}

In recent years, there has been quite a considerable amount of work in models in which glassy behavior is generated not by quenched disorder, but by kinetic constraints. The kinetic restrictions are responsible for the slow relaxation, since the state of a particle or a group of particles can only change if some condition of its environment is fulfilled. In particular, "facilitated" models have been considered, both for structural glasses [1-5] and for granular systems [6]. The characteristic feature of facilitated models is that a particle (spin) can only change its state if a certain number of its neighbors is in an excited state. Also, hierarchically constrained models have been used to study stretched exponential relaxation in glasses [7]. In these models, the system is structured in levels and a particle in a given level can only make a transition if a given cluster of particles in the lower level is in a certain subset of configurations. Then, the dynamics of the several levels are coupled, and the characteristic relaxation times increase with the level index. Hierarchically constrained dynamics may be relevant for those complex systems in which the time evolution of the slowest modes is controlled by the relaxation of the fastest ones. This qualitative picture is adequate to describe, among other problems, proteins relaxation [8] and the densification of powders and structural glasses at high pressure $[9,10]$. Very recently, a kind of hierarchically constrained dynamics has been shown to exhibit, in quite a natural way, logarithmic relaxation [11]. This kind of "anomalous," highly nonexponential, decay is observed in a wide variety of complex systems, including spin glasses [12,13], granular materials $[9,10,14,15]$, structural glasses [16-18], and protein models $[8,19]$.

The aim of this work is to study the dynamical behavior of a general class of hierarchically constrained models when submitted to more complicated processes. In particular, we are interested in the behavior of a system with hierarchical constraints when the temperature changes in time, which makes the coupling between the levels time dependent. The consideration of time-dependent temperature processes requires an extension of the original model as formulated by
Palmer et al. [7]. This will be done in a very simple, but natural, way: the coupling between the levels varies in time because the probability of a cluster configuration allowing relaxation of a particle in the next level depends on the temperature. On the other hand, neither the number of particles in a given level nor the length of the clusters "facilitating" the relaxation depends on the temperature. They are considered as quantities defined in the coarse-grained description of the system introduced to model the physical problem at hand.

Let us note that hierarchical models can also be applied to the analysis of nonthermal systems, such as granular materials in the dense regime. For those materials, thermal energy is not enough to make the system explore the phase space of configurations. Then, the system must be externally excited - for instance, vibrating it - in order to be able to evolve. In these situations, the role of temperature is played by the intensity of the external driving. If the stationary state reached by the system in the long-time limit can be described by Edward's theory [20,21], the compactivity $X$, which is analogy of the temperature in thermal systems, will be a function of the intensity of the external force. Then, by exploiting the analogies of Edward's theory, i.e., substituting volume by energy and compactivity by temperature, it is possible to incorporate nonthermal systems in our formulation.

Processes in which the temperature is time dependent are physically relevant because they can be used to study some characteristic dynamical aspects of glasses. For instance, when a supercooled liquid is cooled down to very low temperatures, a laboratory glass transition is observed. A dramatic change in the behavior of the system takes place, and it departs from the equilibrium curve, getting "frozen" in a far-from-equilibrium state. This transition appears as a consequence of the fast increase of the relaxation time with decreasing temperature. In order to characterize the cooling process, experimental physicists often use the residual value of the relevant physical properties, i.e., the difference between their actual values over the cooling curve and the value obtained by extrapolation of the equilibrium curve to 
the very-low-temperature region $[22,23]$. If the system is reheated from the nonequilibrium state, it returns to equilibrium for high temperatures, but it follows a different curve from the cooling one, giving rise to hysteresis effects. This phenomenon is related to the "nonlinearity" of glassy relaxation: the approach towards the equilibrium curve depends on the configuration of the system, measured by the so-called fictive temperature [22-24]. Narayanaswami's theory provides a phenomenological explanation of this behavior [2225]. Interestingly, a similar behavior has been found in vibrated granular materials when the tapping intensity is varied in a cyclic way [26], although the hysteresis effects are more evident when the heating process begins in a loosely packed state, referred as to the "irreversible" branch in the experiments.

We will start from a very general hierarchical spin model, in which pseudospins are organized into levels, labeled by an index $n$. The pseudospins are assumed to correspond to some coarse-grained description of the system. They can take only two values, representing, for instance, two possible densities of a certain small subvolume of the system. One pseudospin in level $n+1$ can only flip between the two possible values if a cluster of $\mu_{n}$ spins in level $n$ is in a given subset of configurations. This is the basic characteristic of hierarchically constrained models as introduced in Ref. [7], and it slows down the relaxation in level $n+1$, as compared with that of level $n$. Here we will consider the simple choice that all the spins in the cluster must be in the up (excited) state. The exact dynamical equation for the evolution of the pseudospins involves very complicated moments of the probability distribution. To get an exactly solvable model, a "meanfield" approximation will be introduced. Then, the characteristic relaxation time $\tau_{n}$ of level $n$ is seen to increase both with the index label $n$, due to the hierarchical constraint, and also with decreasing temperature, since the configurations allowing the system to relax become less probable when the temperature is lowered.

Some exact dynamical results for systems described with master equations with time-dependent transition rates are known. In particular, the existence of a "normal" solution, i.e., a solution of the master equation that is approached by all the others, has been proved on a very general basis [27]. The main required conditions are the irreducibility of the Markov process for long enough times and that the transition rates be externally controlled, so that they do not depend on the probability distribution of the system. Moreover, it has been established that the normal solution tends to the equilibrium curve for very high temperatures in continuous heating processes [27]. The linear correction of the normal solution with respect to the equilibrium curve has been computed using Hilbert's method [28]. It is not evident whether these results still hold when approximations are introduced in the dynamics of the system. In particular, in "mean-field"'type approximations, the demonstrations in Refs. [27,28] are not valid, since the transition rates become functionals of the probability distribution function. Nevertheless, we will show that all the above mentioned properties apply to our simplified model. This is a good test of the plausibility of the approximations carried out and perhaps an indication that the results obtained here are more general than the derivations in Refs. [27,28].

The organization of the paper is as follows. In Sec. II the hierarchical model is introduced, and the exact evolution equation for the average spin is obtained. By introducing a mean-field approximation, this equation can be closed. Afterwards, a specific, but quite general, choice for the functions defining the model is made. This allows us to introduce a continuous limit in which the relaxation of the system at constant temperature is solved in Sec. III. Time-dependent temperature processes are considered in Sec. IV, where the general solution for the evolution of the probability distribution and the average energy are obtained. The general solution is similar to the expression proposed by Narayanaswami on a phenomenological basis $[22,24,25]$. Section IV is devoted to the analysis of Hilbert's expansion, which is valid in the very-high-temperatures regime. Cooling processes are addressed in Sec. V where, for the sake of simplicity, a concrete cooling law is studied, for which the residual properties are analytically calculated. A qualitative analysis of the glasslike transition is presented in Sec. V. It allows us to give very good estimates of the residual properties and leads to the introduction of the concept of fictive temperature in a very natural way. The behavior of the system when it is reheated from low temperatures is considered in Sec. VI. The main role played by the normal solution for the understanding of the hysteresis effects shows up. Moreover, the analysis clearly indicates that the relevance of the normal solution is not restricted to near equilibrium situations. Finally, a discussion of the main points in this work is given in Sec. VII.

\section{DYNAMICS OF HIERARCHICALLY CONSTRAINED MODELS}

In this section a general kind of spin model with hierarchically constrained dynamics will be introduced. We will focus on the evolution of the average value of the spin, which is supposed to be the relevant variable. For instance, in a thermal system it will be directly related to the mean energy. Then, let us consider a system whose degrees of freedom can be classified into levels, labeled by an index $n$ $=0,1,2, \ldots, n_{\max }$. The degrees of freedom in level $n$ will be represented by $N_{n}$ pseudospins, $\sigma_{i}^{(n)}= \pm 1, i=1,2, \ldots, N_{n}$. The Hamiltonian of the system is assumed to have the form

$$
\mathcal{H}=h \sum_{n=0}^{n_{\max }} \sum_{i=1}^{N_{n}} m_{i}^{(n)}, \quad m_{i}^{(n)}=\frac{1+\sigma_{i}^{(n)}}{2} .
$$

Note that $m_{i}^{(n)}$ is the occupation number of the "up" $(+1)$ state of the corresponding site. In order to write Eq. (2.1) we have supposed that there is no interaction between the pseudospins, but there is an "external field" $h$. For a thermal system, $\mathcal{H}$ gives the energy of a given microstate of the system, while for a nonthermal system, like a powder, it could be interpreted as the volume of a given, mechanically stable, configuration of "grains" [20,21]. Using the terminology for thermal systems, the average value of the dimensionless energy per spin over the ensemble of systems considered is 


$$
\varepsilon=\frac{\langle\mathcal{H}\rangle}{N h}=\frac{1}{N} \sum_{n=0}^{n_{\max }} \sum_{i=1}^{N_{n}} p_{i}^{(n)},
$$

where

$$
p_{i}^{(n)}=\left\langle m_{i}^{(n)}\right\rangle=\frac{1+\left\langle\sigma_{i}^{(n)}\right\rangle}{2}
$$

is the probability that the $i$ th spin of level be in the up state, $N=\sum_{n=0}^{n_{\max }} N_{n}$ is the total number of pseudospins, and the angular brackets denote statistical ensemble average.

The system is considered to be in contact with a heat bath at temperature $T$, so that the equilibrium average value of the pseudospins does not depend either on $i$ nor on $n$, and it is given by

$$
\langle\sigma\rangle_{e} \equiv\left\langle\sigma_{i}^{(n)}\right\rangle_{e}=-\tanh \left(\frac{1}{T^{*}}\right)
$$

Here $T^{*}$ is a dimensionless temperature, and $T^{*}=2 k_{B} T / h$, $k_{B}$ being Boltzmann's constant. For the sake of concision, we will drop the asterisk in the following. The above average value of spin follows from the equilibrium probability for the "up" state of any spin,

$$
p_{e} \equiv p_{i, e}^{(n)}=\frac{e^{-1 / T}}{e^{-1 / T}+e^{1 / T}} .
$$

In the limit of infinite temperature or zero external field, both states of the pseudospins are equiprobable, $p_{e}=1 / 2$, and $\langle\sigma\rangle_{e}=0$. From Eq. (2.2), it follows that the equilibrium value of $\varepsilon$ is

$$
\varepsilon_{e}=p_{e}
$$

For granular materials, the role of the temperature $T$ is played by the compactivity [20,21], which is linked to the intensity of the perturbation, allowing the system to explore the configuration space.

The dynamics of the model is formulated by means of a master equation with single-spin-flip Glauber transition rates [29]. Let us consider the flip of a given spin $\sigma_{i}^{(n)}$. This transition connects a given configuration $\boldsymbol{\sigma}$ of the whole system with the configuration $R_{i}^{(n)} \boldsymbol{\sigma}$, where $R_{i}^{(n)}$ is the operator which rotates the spin $\sigma_{i}^{(n)}$, keeping all the other spins the same. The transition rate for the flip of the spin $\sigma_{i}^{(n)}$ in configuration $\boldsymbol{\sigma}$ is

$$
W_{i}^{(n)}(\boldsymbol{\sigma})=\frac{1}{2} \alpha_{i}^{(n)}(\boldsymbol{\sigma})\left[1+\sigma_{i}^{(n)} \tanh \left(\frac{1}{T}\right)\right]
$$

The characteristic relaxation rate $\alpha_{i}^{(n)}$ of the spin $\sigma_{i}^{(n)}$ depends on the configuration $\boldsymbol{\sigma}$ of the system through the hierarchical constraint

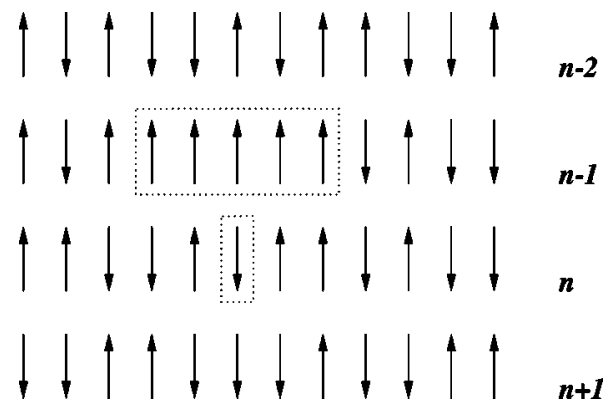

FIG. 1. The framed spin in level $n, \sigma_{i}^{(n)}$, has a nonvanishing probability of changing its state only if the framed cluster of spins in level $n-1$ are in the state shown in the figure, i.e. all of them up $(+1)$. In this example, we have taken $k_{i}=i-2$ and $\mu_{n-1}=5$.

$$
\begin{aligned}
\alpha_{i}^{(n)}(\boldsymbol{\sigma})= & {\left[\alpha_{k_{i}}^{(n-1)}(\boldsymbol{\sigma}) \alpha_{k_{i}+1}^{(n-1)}(\boldsymbol{\sigma}) \cdots \alpha_{k_{i}+\mu_{n-1}-1}^{(n-1)}\right.} \\
& \times(\boldsymbol{\sigma})]^{1 / \mu_{n-1}} \prod_{j=k_{i}}^{k_{i}+\mu_{n-1}-1} \delta_{\sigma_{j}^{(n-1)},+1}
\end{aligned}
$$

where $\delta_{i j}$ is the Kronecker delta. This expression implies that the spin $\sigma_{i}^{(n)}$ needs, in order to flip, that all the spins belonging to a cluster of length $\mu_{n-1}$ starting at a given spin $k_{i}$ of level $n-1$ must be in the up $(+1)$ state. Besides, the characteristic flip rate of the spin is the average of the characteristic flip rates of the spins belonging to the cluster determining its possibility of change. This restricting condition is schematically depicted in Fig. 1. Note that the possibility of a given spin in level $n$ to flip is restricted by the state of a set of clusters in all levels $n^{\prime}<n$, the number of clusters involved in each level increasing as $n^{\prime}$ decreases. The hierarchical constraint implies that the configuration with all the spins in the down $(-1)$ state is completely absorbent; i.e., the system does not evolve in time from that configuration.

We are interested in the time evolution of the average spin $\sigma_{i}^{(n)}$, which is given in Glauber dynamics by [29]

$$
\frac{d}{d t}\left\langle\sigma_{i}^{(n)}\right\rangle=-2\left\langle\sigma_{i}^{(n)} W_{i}^{(n)}(\boldsymbol{\sigma})\right\rangle,
$$

and substitution of Eqs. (2.7) and (2.8) into this expression yields

$$
\begin{aligned}
\frac{d}{d t}\left\langle\sigma_{i}^{(n)}\right\rangle= & -\left\langle\left[\alpha_{k_{i}}^{(n-1)}(\boldsymbol{\sigma}) \alpha_{k_{i}+1}^{(n-1)}(\boldsymbol{\sigma}) \cdots\right.\right. \\
& \left.\times \alpha_{k_{i}+\mu_{n-1}-1}^{(n-1)}(\boldsymbol{\sigma})\right]^{1 / \mu_{n-1}}\left(\sigma_{i}^{(n)}-\langle\sigma\rangle_{e}\right) \\
& \left.\times \prod_{j=k_{i}}^{k_{i}+\mu_{n-1}-1} \delta_{\sigma_{j}^{(n-1)},+1}\right\rangle,
\end{aligned}
$$

where we have taken into account that $\left(\sigma_{i}^{(n)}\right)^{2}=+1$ for all $i$, $n$. This equation is rather involved, since it couples the evolution of $\left\langle\sigma_{i}^{(n)}\right\rangle$ to moments of the probability distribution containing an increasing number of spins of all the levels $n^{\prime}$ such that $0 \leqslant n^{\prime}<n$. The levels $0 \leqslant n^{\prime} \leqslant n-2$ enter into the equation through the rates $\alpha_{j}^{(n-1)}(\boldsymbol{\sigma})$. Then, we introduce at this stage a sort of "mean-field" approximation for the tran- 
sition rates, which is closely related, in spirit, to the seminal work of Palmer et al. [7]. Within this coarsening dynamics, it is reasonable to expect that spins in level $n$ evolve over a time scale quite larger than that characteristic of level $n-1$. This means that spins in level $n-1$ change many times their state before a transition in level $n$ takes place. Thus, we replace the product of the Kronecker deltas in Eq. (2.8) by its average value, i.e., by the probability $P^{(n-1)}\left(\sigma_{k_{i}}^{(n-1)}\right.$ $\left.=+1, \ldots, \sigma_{k_{i}+\mu_{n-1}-1}^{(n-1)}=+1\right)$ that all $\mu_{n-1}$ spins of the given cluster are in the up state,

$$
\begin{aligned}
\alpha_{i}^{(n)}(\boldsymbol{\sigma})= & {\left[\alpha_{k_{i}}^{(n-1)}(\boldsymbol{\sigma}) \alpha_{k_{i}+1}^{(n-1)}(\boldsymbol{\sigma}) \cdots \alpha_{k_{i}+\mu_{n-1}-1}^{(n-1)}(\boldsymbol{\sigma})\right]^{1 / \mu_{n-1}} } \\
& \times P^{(n-1)}\left(\sigma_{k_{i}}^{(n-1)}\right. \\
= & \left.+1, \ldots, \sigma_{k_{i}+\mu_{n-1}-1}^{(n-1)}=+1\right) .
\end{aligned}
$$

Moreover, we will restrict ourselves to situations where there is spatial homogeneity within each of the levels, so that the dependence on the specific site considered in a given level can be dropped, obtaining

$$
\begin{aligned}
\alpha^{(n)}(\boldsymbol{\sigma})= & \alpha^{(n-1)}(\boldsymbol{\sigma}) P^{(n-1)}\left(\sigma_{1}^{(n-1)}=+1, \ldots, \sigma_{\mu_{n-1}}^{(n-1)}=\right. \\
& +1) .
\end{aligned}
$$

Iteration of the above relation gives

$$
\alpha^{(n)}(\boldsymbol{\sigma})=\alpha^{(0)} \prod_{j=0}^{n-1} P^{(j)}\left(\sigma_{1}^{(j)}=+1, \ldots, \sigma_{\mu_{j}}^{(j)}=+1\right),
$$

with $\alpha^{(0)}$ being a constant that characterizes the relaxation rate of the spins belonging to level $n=0$, whose dynamics is not constrained. As $\alpha^{(0)}$ determines the basic time scale, which is arbitrary, we will take $\alpha^{(0)}=1$ in the following. In the mean-field approximation just introduced, the time evolution of the average value of the spin, Eq. (2.10), takes the form

$$
\begin{aligned}
\frac{d}{d t}\left\langle\sigma^{(n)}\right\rangle= & -\prod_{j=0}^{n-1} P\left(\sigma_{1}^{(j)}=+1, \ldots, \sigma_{\mu_{j}}^{(j)}=+1\right)\left(\left\langle\sigma^{(n)}\right\rangle\right. \\
& \left.-\langle\sigma\rangle_{e}\right) .
\end{aligned}
$$

Now, a new approximation will be made. The probability $P^{(j)}$ in Eq. (2.14) will be substituted by its equilibrium value. This would be exact in linear response around equilibrium, but it will be taken here as an approximation leading to the basic equation of our hierarchically constrained model, namely,

$$
\frac{d}{d t}\left\langle\sigma^{(n)}\right\rangle=-\prod_{j=0}^{n-1} p_{e}^{\mu_{j}}\left(\left\langle\sigma^{(n)}\right\rangle-\langle\sigma\rangle_{e}\right)
$$

where $p_{e}$ is the equilibrium probability of any spin being in the up state, given by Eq. (2.5). Again, on physical grounds, this is a sensible approximation due to the separation of the characteristic time scales of the different levels. Because of the hierarchically constrained dynamics, spins in level $n-1$ reach equilibrium over a time scale in which spins in level $n$ have not begun to evolve. However, as a consequence of the last approximation, the configuration with all the spins in the down state is no longer absorbent.

From Eq. (2.15), an equivalent equation can be written for the evolution of the probability $p^{(n)}$ of the up state in level $n$, defined in Eq. (2.3), i.e.,

$$
\frac{d}{d t} p^{(n)}=-\alpha_{n}\left(p^{(n)}-p_{e}\right)
$$

$\alpha_{n}$ being the characteristic relaxation rate of level $n$,

$$
\alpha_{n}=p_{e}^{g_{n}}, \quad g_{n}=\sum_{j=0}^{n-1} \mu_{j}
$$

Equation (2.16) implies that, due to the hierarchical constraints, the spin relaxation slows down with increasing level $n$, since $\alpha_{n}$ is a decreasing function of $n$, because $p_{e}<1$. Equation (2.16) is the main result in this section. In the following, we will explore its implications, considering first processes at constant temperature in the next section, and cooling and heating processes in the remainder of the paper.

\section{RELAXATION AT CONSTANT TEMPERATURE}

For the case of constant temperature $T$, and therefore constant $\alpha_{n}$, Eq. (2.16) is easily solved,

$$
p^{(n)}(t)-p_{e}=\left[p^{(n)}(0)-p_{e}\right] e^{-\alpha_{n} t} .
$$

Then, each spin relaxes exponentially to equilibrium with the rate characteristic of its level.

For the homogenous situations within each level we are considering, the dimensionless mean energy per spin [30] defined in Eq. (2.2) simplifies to

$$
\varepsilon(t)=\sum_{n=0}^{n_{\max }} w_{n} p^{(n)}(t)
$$

where $w_{n}=N_{n} / N$ is the fraction of spins in level $n$, verifying $\sum_{n=0}^{n_{\max }} w_{n}=1$.

Putting Eq. (3.1) into Eq. (3.2) yields

$$
\varepsilon(t)=\varepsilon_{e}(T)+\sum_{n=0}^{n_{\max }} w_{n}\left[p^{(n)}(0)-p_{e}\right] e^{-\alpha_{n} t},
$$

for the relaxation of the energy at constant temperature. In order to proceed, we will consider the simple case in which the initial probability distribution $p^{(n)}(0)$ does not depend on the index level $n$. This will be the situation, for instance, when the initial state corresponds to equilibrium at a different temperature $T+\Delta T$. Thus, the relaxation function of the physical property described by the Hamiltonian of the system is given by

$$
\phi(t) \equiv \frac{\varepsilon(t)-\varepsilon_{e}}{\varepsilon(0)-\varepsilon_{e}}=\sum_{n=0}^{n_{\max }} w_{n} e^{-\alpha_{n} t} .
$$


This equation is a generalization of the result derived by Palmer et al. in their pioneering work in hierarchically constrained dynamics [7], which corresponds to the choice $p_{e}$ $=1 / 2$. This is formally equivalent to the particularization of Eq. (3.4) for $T \rightarrow \infty$. If other positive values of the temperature are considered, the effect is an increase of the relaxation times

$$
\tau_{n}=\alpha_{n}^{-1}
$$

since $p_{e}$ is a decreasing function of the temperature. A main advantage of the formulation of the hierarchical models as presented here, aside from its larger generality, is that it allows analysis of processes in which the temperature of a thermal system (or the vibration intensity in a granular system) changes in time. This kind of processes will be addressed in the next section. A mean relaxation time $\tau$ can be defined as

$$
\tau=\int_{0}^{\infty} d t \phi(t)=\sum_{n=0}^{n_{\max }} w_{n} \tau_{n},
$$

providing a quantitative measure of the time it takes the system to relax to equilibrium at temperature $T$.

Let us consider that the fraction of spins in level $n, w_{n}$, and the number of "facilitating" spins in level $n, \mu_{n}$, depend very smoothly on $n$; i.e., they can be expressed as functions of the form

$$
w_{n}=w(n \eta) \quad \mu_{n}=\mu(n \eta),
$$

where $\eta \ll 1$. These seem to be sensible conditions when modeling a real system, in which the introduction of the levels and the pseudospins is associated to some coarsegrained description. By defining

$$
x_{n}=n \eta
$$

which is a continuous variable in the limit $\eta \rightarrow 0$, the sums over $n$ can be replaced by integrals. The relaxation rate of level $n$, given by Eq. (2.17), becomes a function of the continuous variable $x$,

$$
\alpha(x)=p_{e}^{g(x)},
$$

with

$$
g(x)=\frac{\sum_{k=0}^{n-1} \mu_{k} \eta}{\sum_{k=0}^{n} w_{k} \eta}=\frac{\int_{0}^{x} d x^{\prime} \mu\left(x^{\prime}\right)}{\int_{0}^{x_{\max }} d x^{\prime} w\left(x^{\prime}\right)},
$$

where $x_{\max }=n_{\max } \eta$, and the normalization of the weights $w_{n}$ has been used. Therefore, the relaxation function $\phi(t)$, given by Eq. (3.4), becomes

$$
\phi(t)=\frac{\sum_{n=0}^{n_{\max }} \eta w_{n} e^{-\alpha_{n} t}}{\sum_{n=0}^{n_{\max }} \eta w_{n}}=\frac{\int_{0}^{x_{\max }} d x w(x) e^{-\alpha(x) t}}{\int_{0}^{x_{\max }} d x w(x)},
$$

in the continuous limit.

In general, Eq. (3.11) is mathematically rather involved, since it depends both on the functions $\mu(x)$ and $w(x)$. The simplest possibility appears to be $\mu(x)$ proportional to $w(x)$, i.e., $\mu_{n}$ proportional to $w_{n}$ or, equivalently, to $N_{n}$. In other words, the simplest kind of hierarchically constrained models shows up when the number of "facilitating" spins at a level is an extensive function of the number of spins at the same level [11]. This condition is expressed as

$$
\mu(x)=a w(x),
$$

with $a$ being a constant, independent of $x$. In this case, it is useful to define the new variable

$$
u=\frac{\int_{0}^{x} d x^{\prime} w\left(x^{\prime}\right)}{\int_{0}^{x_{\max }} d x^{\prime} w\left(x^{\prime}\right)},
$$

measuring the fraction of spins belonging to levels up to $n$ $=x / \eta$. In terms of $u$, the relaxation rates of Eq. (3.9) are given by

$$
\alpha(u)=p_{e}^{g(u)}, \quad g(u)=a u,
$$

the relaxation function is expressed as

$$
\phi(t)=\int_{0}^{1} d u e^{-\alpha(u) t},
$$

and the mean relaxation time reads

$$
\tau=\int_{0}^{\infty} d t \phi(t)=\int_{0}^{1} d u \tau(u)=\frac{p_{e}^{-a}-1}{a\left|\ln p_{e}\right|},
$$

with $\tau(u)=\alpha^{-1}(u)$. It is interesting to consider situations for which $p_{e}^{a} \ll 1$, so that the minimum relaxation rate $\alpha(1)$ is much smaller than the maximum one $\alpha(0)=1$ in our dimensionless time scale. In this case, the relaxation function $\phi(t)$ is linear in $\ln t$ over an intermediate time window, 1 $\ll t \ll p_{e}^{-a}$, namely [11],

$$
\phi(t) \sim 1-\frac{1}{a\left|\ln p_{e}\right|}(\gamma+\ln t),
$$

where $\gamma$ stands for Euler's constant, $\gamma \simeq 0.577$. This kind of linear logarithmic behavior is characteristic of a great variety of complex systems, including spin glasses [12,13], granular materials $[9,10,14,15]$, structural glasses [16-18], and protein models $[8,19]$. In the present context, the condition $p_{e}^{a}$ $\ll 1$ corresponds to a "low"-temperature limit, in which the 
mean relaxation time, given by Eq. (3.16), is very large; i.e., the relaxation of the system becomes very slow.

It is worth noting that, in terms of the $u$ variable, the continuous limit is formally obtained by changing the functions of the index level $n$ by the corresponding functions of the variable $u$ and by making the replacement

$$
\sum_{n=0}^{n_{\max }} w_{n} \rightarrow \int_{0}^{1} d u
$$

It follows that, in the continuous limit, the dynamical behavior of the system does not depend explicitly on the level populations $N_{n}$, but only on the relaxation rates $\alpha$ expressed as functions of $u$, as given by Eq. (3.14).

\section{TIME-DEPENDENT TEMPERATURE PROCESSES}

In this section processes in which the temperature changes in time will be studied. The evolution equation (2.16) is now

$$
\frac{d}{d t} p^{(n)}(t)=-\alpha_{n}(T)\left[p^{(n)}(t)-p_{e}(T)\right],
$$

where $T=T(t)$. The general solution of Eq. (4.1) is

$$
\begin{aligned}
p^{(n)}(t)= & {\left[p^{(n)}\left(t_{0}\right)-p_{e}\left(T_{0}\right)\right] \chi_{n}\left(t, t_{0}\right)+p_{e}(T) } \\
& -\int_{t_{0}}^{t} d t^{\prime} \frac{d p_{e}\left(T^{\prime}\right)}{d T^{\prime}} \frac{d T^{\prime}}{d t^{\prime}} \chi_{n}\left(t, t^{\prime}\right) .
\end{aligned}
$$

Here $T_{0}=T\left(t_{0}\right)$ is the initial value of the temperature, $T$ $=T(t), T^{\prime}=T\left(t^{\prime}\right), T^{\prime \prime}=T\left(t^{\prime \prime}\right)$, and we have introduced the function

$$
\chi_{n}\left(t_{1}, t_{2}\right)=\exp \left(-\int_{t_{2}}^{t_{1}} d t \alpha_{n}(t)\right)
$$

The above equation is valid for any law of variation for the temperature. Taking into account Eq. (3.2), the average energy per spin, $\varepsilon$, is given by [30]

$$
\begin{aligned}
\varepsilon(t)= & \sum_{n=0}^{n_{\max }} w_{n}\left[p^{(n)}\left(t_{0}\right)-p_{e}\left(T_{0}\right)\right] \chi_{n}\left(t, t_{0}\right)+\varepsilon_{e}(T) \\
& -\int_{t_{0}}^{t} d t^{\prime} \frac{d \varepsilon_{e}\left(T^{\prime}\right)}{d T^{\prime}} \frac{d T^{\prime}}{d t^{\prime}} \sum_{n=0}^{n_{\max }} w_{n} \chi_{n}\left(t, t^{\prime}\right),
\end{aligned}
$$

where $\varepsilon_{e}$ is the average equilibrium energy, defined in Eq. (2.6).

Let us assume that the system is initially at equilibrium with $T=T_{0}$. Then the first term on the right-hand side (RHS) of Eq. (4.4) vanishes and

$$
\varepsilon(t)=\varepsilon_{e}(T)-\int_{t_{0}}^{t} d t^{\prime} \frac{d \varepsilon_{e}\left(T^{\prime}\right)}{d T^{\prime}} \frac{d T^{\prime}}{d t^{\prime}} M\left(t, t^{\prime}\right),
$$

where

$$
M\left(t, t^{\prime}\right)=\sum_{n=0}^{n_{\max }} w_{n} \chi_{n}\left(t, t^{\prime}\right)
$$

is a memory function. In the case of constant temperature, $M\left(t, t^{\prime}\right)$ is equal to the relaxation function $\phi\left(t-t^{\prime}\right)$, as seen by comparing Eq. (4.6) with Eq. (3.4). The structure of Eq. (4.5) is the same as that of Narayanaswami's phenomenological theory of glasses [22-25]. A similar result was obtained some years ago for the one-dimensional Ising model with Glauber dynamics [31].

\section{High-temperature limit: Hilbert's method}

We are going to look for a solution of Eq. (4.1) by means of Hilbert's method. A special solution

$$
p_{H}^{(n)}(t)=\sum_{k=0}^{\infty} p_{H}^{(n), k}(t)
$$

is constructed in an iterative way as follows. We take

$$
p_{H}^{(n), 0}(t)=p_{e}(T)
$$

while for $k \geqslant 1$

$$
p_{H}^{(n), k}(t)=-\alpha_{n}^{-1}(T) \frac{d p^{(n), k-1}(t)}{d t} .
$$

Equation (4.8) shows that Hilbert's expansion agrees with the equilibrium distribution to the lowest order. Besides, for $k=1$ we get from Eq. (4.9)

$$
p_{H}^{(n), 1}(t)=-\tau_{n}(T) \frac{d p_{e}(T)}{d T} \frac{d T}{d t} .
$$

This equation indicates the main limitation of Hilbert's method. Due to the divergence of the relaxation times $\tau_{n}$ $=\alpha_{n}^{-1}$ in the low-temperature limit [see Eq. (2.17)], also $p^{(n), 1}$ diverges in that limit. As a consequence, Hilbert's solution is only accurate in the high-temperature regime, in which an expansion around equilibrium provides a good approximation. Restricting ourselves to high temperatures, we approximate

$$
p_{H}^{(n)}(T) \simeq p_{e}(T)-\tau_{n}(T) \frac{d p_{e}(T)}{d T} \frac{d T}{d t}
$$

and, from Eq. (3.2),

$$
\varepsilon_{H}(T) \simeq \varepsilon_{e}(T)-\frac{d \varepsilon_{e}(T)}{d T} \frac{d T}{d t} \sum_{n=0}^{n_{\max }} w_{n} \tau_{n}(T) .
$$

Taking into account the definition of the average relaxation time $\tau$, Eq. (3.6), the above expression is seen to be equivalent to

$$
\varepsilon_{H}(T) \simeq \varepsilon_{e}(T)-\frac{d \varepsilon_{e}(T)}{d T} \frac{d T}{d t} \tau(T),
$$


which agrees with the high-temperature behavior of Eq. (4.5). In Eqs. (4.11) and (4.13), $p_{H}^{(n)}$ and $\varepsilon_{H}$ depend on time only through the temperature $T(t)$. Thus, Hilbert's method provides a "normal" solution, in the sense often used in kinetic theory. The validity of a expression identical to Eq. (4.13), also in the high-temperature limit, has been established for a quite general class of systems whose dynamics is described by a master equation [28]. Although we have made here several drastic approximations in order to get a closed equation for the average spin, the high-temperature limit of the solution, given by Hilbert's method, remains formally the same as that of the exact solution of the original model. Certainly, this is a good property of those approximations.

What is the physical meaning of the failure of Hilbert's expansion for low temperatures? Due to the divergence of the characteristic relaxation times, the system does not have enough time to relax to the equilibrium curve at very low temperatures, and it gets "frozen" in a far-from-equilibrium state. Since Hilbert's method is an expansion around equilibrium, it fails in the low-temperature region. In fact, the RHS of Eq. (4.11) becomes negative for low enough temperatures. On the other hand, Hilbert's expansion is useful to estimate the values of the physical properties in the "frozen" state [28]. Also, Hilbert's method provides a qualitative understanding of the hysteresis effects appearing in thermal cycles (cooling and reheating). In cooling processes $(d T / d t<0)$, it is $\varepsilon_{H} \geqslant \varepsilon_{e}$, while in heating processes $(d T / d t>0)$, it is $\varepsilon_{H}$ $\geqslant \varepsilon_{e}$. Then, $\varepsilon_{H}$ lies to opposite sides of the equilibrium curve for cooling and heating processes, and hysteresis effects show up in thermal cycling experiments, as will be discussed in more detail in Sec. VI.

\section{COOLING PROCESSES}

Next, we are going to study the continuous cooling of the system down to very low temperatures. The origin of time is taken at the beginning of the cooling process. The initial condition will be the equilibrium configuration at a "high" temperature $T_{0}$, i.e.,

$$
p^{(n)}(0)=p_{e}\left(T_{0}\right)
$$

Then, the first correction in Hilbert's expansion, $p^{(n), 1}(t)$, is very small as compared with $p_{e}\left(T_{0}\right)$ for $T \rightarrow T_{0}$. Particularization of Eq. (4.2) for the above initial condition gives

$$
p^{(n)}(t)=p_{e}(T)-\int_{0}^{t} d t^{\prime} \frac{d p_{e}\left(T^{\prime}\right)}{d T^{\prime}} \frac{d T^{\prime}}{d t^{\prime}} \chi_{n}\left(t, t^{\prime}\right)
$$

Since $p_{e}(T)$ is an increasing function of the temperature, for continuous cooling processes it is

$$
p^{(n)}(t) \geqslant p_{e}(T) \quad \text { for all } t \text { and } n
$$

The possible deviations from the equilibrium distribution always lead to an increase of the probability of the spin being in the excited state. Moreover, Eq. (5.2) directly implies that
$\varepsilon(t)=\varepsilon_{e}(T)-\int_{0}^{t} d t^{\prime} \frac{d \varepsilon_{e}\left(T^{\prime}\right)}{d T^{\prime}} \frac{d T^{\prime}}{d t^{\prime}} \sum_{n=0}^{n_{\max }} w_{n} \chi_{n}\left(t, t^{\prime}\right) \geqslant \varepsilon_{e}(T)$,

where we have used Eq. (3.2). This inequality has been experimentally observed in glass-forming liquids [22,23]. Since the reported experiments were made at constant pressure, the quantity $\varepsilon$ considered here must be interpreted as the enthalpy in that context.

In order to proceed further in our analysis, the continuous limit introduced in the study of the relaxation at constant temperature in Sec. III will be considered. As already mentioned, this continuous limit is expected to be closer to the description of real systems than the discrete level picture. Besides, for the sake of concreteness, we will restrict ourselves to those models verifying Eq. (3.12). The index level $n$ is substituted by the continuous variable $u$, defined in Eq. (3.13), representing the fraction of the total number of spins up to level $n$. With an obvious change of notation, Eq. (5.2) becomes

$$
p(t ; u)=p_{e}(T)-\int_{0}^{t} d t^{\prime} \frac{d p_{e}\left(T^{\prime}\right)}{d T^{\prime}} \frac{d T^{\prime}}{d t^{\prime}} \chi\left(t, t^{\prime} ; u\right),
$$

where

$$
\chi\left(t, t^{\prime} ; u\right)=\exp \left(-\int_{t^{\prime}}^{t} d t^{\prime \prime} \alpha\left(T^{\prime \prime} ; u\right)\right)
$$

with $\alpha(T ; u)$ given by Eq. (3.14), i.e.,

$$
\alpha(T ; u)=p_{e}(T)^{a u} .
$$

Also, using Eq. (3.18), it is found that

$$
\varepsilon(t)=\varepsilon_{e}(T)-\int_{0}^{t} d t^{\prime} \frac{d \varepsilon_{e}\left(T^{\prime}\right)}{d T^{\prime}} \frac{d T^{\prime}}{d t^{\prime}} \int_{0}^{1} d u \chi\left(t, t^{\prime} ; u\right) .
$$

The time evolution of the probability $p(t ; u)$ depends on the explicit form of the cooling law. In experiments, linear cooling is usually employed,

$$
\frac{d T}{d t}=-r_{c}
$$

where $r_{c}>0$ is the cooling rate determining the time scale $r_{c}^{-1}$ over which the temperature changes. Linear cooling implies that $p_{e}(T)$ depends on time in a rather involved way. From Eqs. (2.5) and (5.9) one gets

$$
\frac{d p_{e}}{d t}=-\frac{1}{2} r_{c} p_{e}\left(1-p_{e}\right)\left(\ln \frac{p_{e}}{1-p_{e}}\right)^{2} .
$$

We are interested in cooling processes for which the temperature changes slowly in time, $r_{c} \ll 1$, so that the system departs from the equilibrium curve for very low temperatures, where $p_{e} \ll 1$. Then, a law equivalent to linear cooling, aside from logarithmic corrections, is 


$$
\frac{d p_{e}}{d t}=-r_{c} p_{e}
$$

having the advantage that analytical calculations are much more simple with Eq. (5.12) [31-33]. In the following, we will use the notation

$$
p_{e}(T)=p_{e}, \quad p_{e}\left(T^{\prime}\right)=p_{e}^{\prime}, \quad p_{e}\left(T_{0}\right)=p_{e 0} .
$$

The time integrals in Eq. (5.5) can be transformed into integrals over $p_{e}$ by means of the cooling law (5.11), with the result

$$
p(t ; u)=p_{e}+\int_{p_{e}}^{p_{e 0}} d p_{e}^{\prime} \exp \left(-\frac{p_{e}^{\prime a u}-p_{e}^{a u}}{r_{c} a u}\right) .
$$

The second term in this expression is dominant in the lowtemperature region, where $p_{e}$ is very small. Therefore, it follows that spins in any level $u$ fall in a nonequilibrium state for low enough temperatures. The details of this glasslike transition will be analyzed below, in a separate subsection.

One of the main quantities characterizing a given cooling process is the residual value $f_{\text {res }}$ of a relevant property $f$. The residual value measures the excess with respect to the equilibrium curve, extrapolated to very low temperatures,

$$
f_{\text {res }}=\lim _{T \rightarrow 0}\left(f-f_{e}\right) .
$$

In particular, for the probability $p(t ; u)$ we have from Eq. (5.13)

$$
p_{\text {res }}(u)=\int_{0}^{p_{e 0}} d p_{e}^{\prime} \exp \left(-\frac{p_{e}^{\prime a u}}{r_{c} a u}\right)
$$

which is easily transformed into

$$
p_{\text {res }}(u)=\frac{1}{a u}\left(r_{c} a u\right)^{\frac{1}{a u}} \int_{0}^{x_{0}} d x x^{\frac{1}{a u}-1} e^{-x},
$$

where $x_{0}=p_{e 0}^{a u} /\left(r_{c} a u\right)$. The slow cooling limit is defined by the residual properties being independent of the initial conditions and determined univocally by the cooling rate [3134]. In our case, slow cooling means that the upper integration limit in Eq. (5.16) can be substituted by infinity for all $u$, i.e.,

$$
\frac{p_{e 0}^{a u}}{r_{c} a u} \gg 1
$$

As the $u$ variable varies in the interval $0 \leqslant u \leqslant 1$, the slow cooling condition is

$$
r_{c} a \ll p_{e 0}^{a}<1 .
$$

Then, with an exponentially small error,

$$
p_{\text {res }}(u) \sim \frac{1}{a u}\left(r_{c} a u\right)^{1 / a u} \Gamma\left(\frac{1}{a u}\right)=\left(r_{c} a u\right)^{1 / a u} \Gamma\left(1+\frac{1}{a u}\right) .
$$

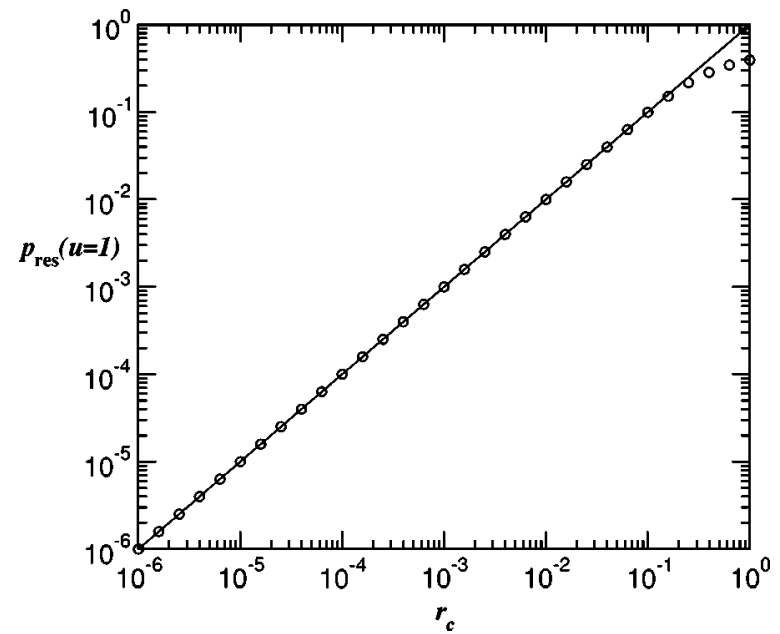

FIG. 2. Residual probability for the slowest modes $p_{\text {res }}(u=1)$ as a function of the dimensionless cooling rate $r_{c}$ defined in the main text. The circles correspond to the numerical integration of Eq. (5.16), while the solid line is the prediction of the asymptotic calculation, Eq. (5.19). A good agreement is observed up to $r_{c} \simeq 0.1$.

This expression gives the probability that the spins in level $u$ be in the up state at very low temperatures. In Fig. 2, the residual probability $p_{\text {res }}(u=1)$ is plotted as a function of the cooling rate $r_{c}$, for $a=1$. The asymptotic result, given by Eq. (5.19), is compared with the numerical integration of Eq. (5.16) with $p_{e 0}=1 / 2$; i.e., the system is taken initially at infinite temperature. The agreement is quite good up to $r_{c}$ $\simeq 0.1$, which is not very small.

The evolution of the average energy, for the cooling law (5.11), is given by

$$
\begin{aligned}
\varepsilon(t)-\varepsilon_{e}(T) & =\int_{0}^{1} d u\left[p(t ; u)-p_{e}\right] \\
& =\int_{0}^{1} d u \int_{p_{e}}^{p_{e} 0} d p_{e}^{\prime} \exp \left(-\frac{p_{e}^{\prime a u}-p_{e}^{a u}}{r_{c} a u}\right)
\end{aligned}
$$

The residual energy can be easily computed by particularizing this expression for $T \rightarrow 0$,

$$
\varepsilon_{\mathrm{res}}=\int_{0}^{1} d u p_{\mathrm{res}}(u)
$$

The integrand $p_{\text {res }}(u)$, given by Eq. (5.19), vanishes exponentially in the limit $u \rightarrow 0$. A standard Laplace analysis can be made, with the result

$$
\varepsilon_{\mathrm{res}} \sim \Gamma\left(1+\frac{1}{a}\right) \frac{\left(r_{c} a\right)^{1 / a}}{\left|\ln \left(r_{c} a\right)^{1 / a}\right|} .
$$

The leading behavior is potential with $r_{c}$, since

$$
\ln \varepsilon_{\mathrm{res}} \sim \frac{1}{a} \ln \left(r_{c} a\right),
$$




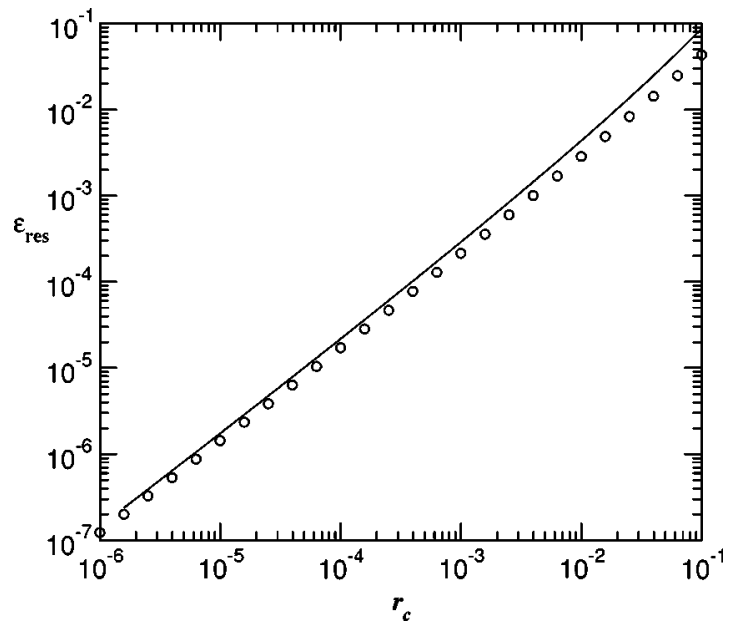

FIG. 3. Dimensionless residual energy $\varepsilon_{\text {res }}$ as a function of the cooling rate $r_{c}$. As in Fig. 2, the circles are from the numerical integration of Eq. (5.16) and the solid line is the prediction of the asymptotic analysis, given by Eq. (5.22). The agreement is good for $r_{c} \lesssim 0.01$.

which comes from the upper limit of integration, $u=1$, corresponding to the largest relaxation time. The integral over the whole distribution function $p_{\text {res }}(u)$ gives a logarithmic correction $\left|\ln \left(r_{c} a\right)\right|^{-1}$, which makes the residual energy smaller than the dominant term $\left(r_{c} a\right)^{1 / a}$. This is due to the increasing behavior of $p_{\text {res }}(u)$ with $u, p_{\text {res }}(u) \leqslant p_{\text {res }}(u=1)$. In Fig. 3 the residual value of the energy is plotted. The asymptotic expression, Eq. (5.22), is compared with the numerical results from Eqs. (5.21) and (5.16). Good agreement is found up to $r_{c}=0.01$. It is worth noting that, for the cooling law considered, the logarithmic correction to the potential in $r_{c}$ behavior is not present for other simple models of structural glasses previously studied [31-35].

\section{Demarcation mode, fictive temperature, and glass transition}

The existence of nonvanishing residual properties is an indication of the departure of the system from equilibrium at low temperatures. Due to the divergence of the characteristic relaxation times $\tau_{n}$, for low enough temperatures the system does not have enough time to relax towards equilibrium, and a kinetic phenomenon resembling the laboratory glass transition [22-24] shows up. Next, we will try to understand the physical origin of this kinetic transition.

Let us consider again the time evolution of the probability distribution $p(t ; u)$ in a cooling process, as given by Eqs. (5.5) and (5.6). The integral in $\chi\left(t, t^{\prime} ; u\right)$,

$$
I\left(t, t^{\prime} ; u\right)=\int_{t^{\prime}}^{t} d t^{\prime \prime} \alpha\left(T^{\prime \prime} ; u\right),
$$

is a measure of the average number of transitions occurring in level $u$ in the time interval between $t^{\prime}$ and $t$. Consequently, a mathematical definition for the limit of slow cooling is that the condition

$$
I\left(t^{*}, 0 ; u\right) \gg 1
$$

hold for all $u$, where $t^{*}$ is the time for which the temperature vanishes if extrapolated accordingly to the prescribed cooling law, i.e., $T\left(t^{*}\right)=0$. Equation (5.25) guarantees that the system experiments a large number of transitions before getting eventually frozen, so that it has enough time to forget the details of the initial condition. For the cooling law defined in Eq. (5.11) it is easily verified that Eq. (5.25) is equivalent to Eq. (5.17).

If we are dealing with a slow cooling process, Eq. (5.25) implies that there is a time window over which

$$
I(t, 0 ; u) \gg 1 .
$$

This is the time regime we are interested in. Let us analyze the behavior of $\chi\left(t, t^{\prime} ; u\right)$ as a function of $t^{\prime}, t_{0} \leqslant t^{\prime} \leqslant t$, for a given time $t$ such that Eq. (5.26) holds. The function $I\left(t, t^{\prime} ; u\right)$ changes from a very large value to zero when $t^{\prime}$ goes from 0 to $t$. Consequently, $\chi\left(t, t^{\prime} ; u\right)$ increases from practically zero to unity when $t^{\prime}$ moves in the above time interval. Let us define a time $t_{f}(t ; u)$, prior to $t$, by

$$
I\left(t, t_{f} ; u\right)=1
$$

so that the average number of transitions taking place in level $u$ in the time interval between $t_{f}$ and $t$ equals unity. Then, $\chi\left(t, t_{f} ; u\right)=e^{-1}$, and the function $\chi\left(t, t^{\prime} ; u\right)$ changes from zero to unity in a certain time interval around $t_{f}$. In order to proceed, we will assume that this change takes place in the vicinity of $t_{f}$ very rapidly, as compared with the variation of the rest of the integrand of Eq. (5.5). Decomposing Eq. (5.5) in the form

$$
\begin{aligned}
p(t ; u)= & p_{e}(T)-\int_{0}^{t_{f}} d t^{\prime} \frac{d p_{e}\left(T^{\prime}\right)}{d T^{\prime}} \frac{d T^{\prime}}{d t^{\prime}} \chi\left(t, t^{\prime} ; u\right) \\
& -\int_{t_{f}}^{t} d t^{\prime} \frac{d p_{e}\left(T^{\prime}\right)}{d T^{\prime}} \frac{d T^{\prime}}{d t^{\prime}} \chi\left(t, t^{\prime} ; u\right),
\end{aligned}
$$

the first integral is subdominant with respect to the second one and, moreover,

$$
\begin{aligned}
\int_{t_{f}}^{t} d t^{\prime} \frac{d p_{e}\left(T^{\prime}\right)}{d T^{\prime}} \frac{d T^{\prime}}{d t^{\prime}} \chi\left(t, t^{\prime} ; u\right) & \simeq \int_{t_{f}}^{t} d t^{\prime} \frac{d p_{e}\left(T^{\prime}\right)}{d T^{\prime}} \frac{d T^{\prime}}{d t^{\prime}} \\
& =p_{e}(T)-p_{e}\left[T_{f}(t ; u)\right],
\end{aligned}
$$

where $T_{f}(t ; u)$ is the temperature of the system at time $t_{f}(t ; u)$, i.e.,

$$
T_{f}(t ; u)=T\left[t_{f}(t ; u)\right] .
$$

Note that $T_{f}(t ; u)>T$, since the time instant $t_{f}(t ; u)<t$. Substitution of Eq. (5.29) into Eq. (5.28) and use of the above approximations yields

$$
p(t ; u)=p_{e}\left[T_{f}(t ; u)\right] .
$$

The arguments leading from Eq. (5.5) to Eq. (5.31) are formally equivalent to assume that 


$$
\chi\left(t, t^{\prime} ; u\right)=\Theta\left[t^{\prime}-t_{f}(t ; u)\right],
$$

where $\Theta(x)$ is Heaviside's step function. The result in Eq. (5.31) has a neat physical interpretation: the probability distribution of spins in level $u$ at time $t$ is given by the equilibrium distribution corresponding to the temperature of the system at a prior time $t_{f}(t ; u)$, defined in Eq. (5.27). The temperature $T_{f}(t ; u)$ is, therefore, the "fictive" temperature of level $u$ for the cooling program under consideration, in the sense used in the phenomenological theories of glassforming liquids [22-24]. In the present context, each level has its own fictive temperature, so that there is not a unique equilibrium distribution describing the whole state of the system at a given temperature. As a consequence, the behavior of different macroscopic properties of the system may be quite different, depending on the modes which dominate for the evaluation of each property.

The definition of the fictive temperature, Eq. (5.30), can be written as

$$
I\left(t^{*}, t_{f} ; u\right)=I\left(t^{*}, t ; u\right)+I\left(t, t_{f} ; u\right)=I\left(t^{*}, t ; u\right)+1
$$

In the "high-temperature" regime, where the number of transitions between temperature $T$ and $T=0$ in level $u$ is very large, we can neglect unity on the RHS of Eq. (5.33) and we get $T_{f}(t ; u) \simeq T$; the modes in level $u$ remain in equilibrium. On the other hand, in the "low-temperature" limit, $I\left(t^{*}, t ; u\right)$ becomes very small and $T_{f}(t ; u)$ tends to a limiting value,

$$
T_{f}^{*}(u)=\lim _{t \rightarrow t^{*}} T_{f}(t ; u)=T\left[t_{f}^{*}(u)\right],
$$

where $t_{f}^{*}(u)$ is the time for which

$$
I\left(t^{*}, t_{f}^{*} ; u\right)=\int_{t_{f}^{*}}^{t^{*}} d t \alpha(T ; u)=1 .
$$

The low-temperature region for level $u$ corresponds to temperatures such that the average number of transitions remaining to spins in level $u$ before formally reaching $T=0$ is smaller than unity. Since the rate $\alpha(T ; u)$ decreases as a function of $u$ [see Eq. (3.14)], $T_{f}^{*}(u)$ is an increasing function of $u$; i.e., the fictive temperature $T_{f}^{*}(u)$ is larger the slower the modes. Then, the following picture of the kinetic glasslike transition appears. The relaxation modes associated with level $u$ are in equilibrium for $T \gtrsim T_{f}^{*}(u)$, getting frozen in their equilibrium distribution corresponding to the fictive temperature $T_{f}^{*}(u)$ for $T \lesssim T_{f}^{*}(u)$. A similar idea was first used in the context of the structural glass transition by Dyre [36], who characterized the transition by means of the concept of demarcation mode. For a given value of the temperature $T$, the modes in levels with $T_{f}^{*}(u)>T$ will be frozen, while those with $T_{f}^{*}(u)<T$ will still be able to relax. The demarcation mode at temperature $T, u_{d}(T)$, is defined by $T_{f}^{*}\left(u_{d}\right)=T$. The modes with $u>u_{d}(T)$ are frozen at this temperature, and modes with $u<u_{d}(T)$ can still evolve. The glass transition can be understood as the movement of the demarcation mode from $u=1$, corresponding to the largest relaxation time, to $u=0$, which is the fastest mode. The tem-

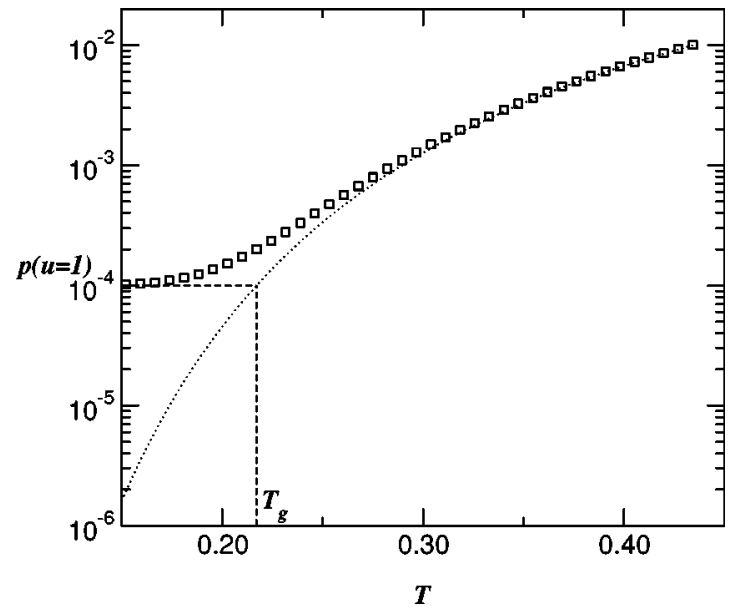

FIG. 4. Probability for the slowest level, $p(u=1)$, as a function of the dimensionless temperature in a process with the cooling law of Eq. (5.11) and a cooling rate $r_{c}=10^{-4}$ (squares). The glass transition temperature $T_{g}$, as given by Eq. (5.39), is indicated. It gives a good estimate of the actual fictive temperature for this level. The equilibrium probability $p_{e}$, Eq. (2.5), is also plotted (dotted line).

perature $T_{g}$ at which the glass transition begins for a given cooling program is that for which the slowest modes freeze, i.e., $T_{g}=T_{f}^{*}(u=1)$.

The physical image developed in the above paragraph allows us to estimate the residual values in quite a simple way. For a given cooling law, first we calculate the limit value of the fictive temperature $T_{f}^{*}(u)$. Then, the residual probability distribution is given by

$$
p_{\text {res }}(u) \simeq p_{e}\left[T_{f}^{*}(u)\right],
$$

and, consequently, the residual value of the energy is

$$
\varepsilon_{\mathrm{res}}=\int_{0}^{1} d u p_{\mathrm{res}}(u) \simeq \int_{0}^{1} d u p_{e}\left[T_{f}^{*}(u)\right] .
$$

As a test, we have considered the cooling process of the hierarchical model studied in this paper with the law given by Eq. (5.11). In the slow-cooling limit, it is easy to show that

$$
T_{f}^{*}(u) \simeq \frac{2 a u}{\left|\ln \left(r_{c} a\right)\right|}
$$

i.e., the glasslike transition begins at

$$
T_{g} \simeq \frac{2 a}{\left|\ln \left(r_{c} a\right)\right|},
$$

for which the slowest modes become frozen. In Fig. 4 we plot the evolution of the probability, as given by Eq. (5.13), of the slowest level $u=1$, for $a=1$ and $r_{c}=10^{-4}$. For these parameters, it is $T_{g}=T_{f}^{*}(u=1) \simeq 0.217$, which is seen to be a good value for the fictive temperature. It is worth noting that the simple arguments used in this section lead to an estimate of the residual population 


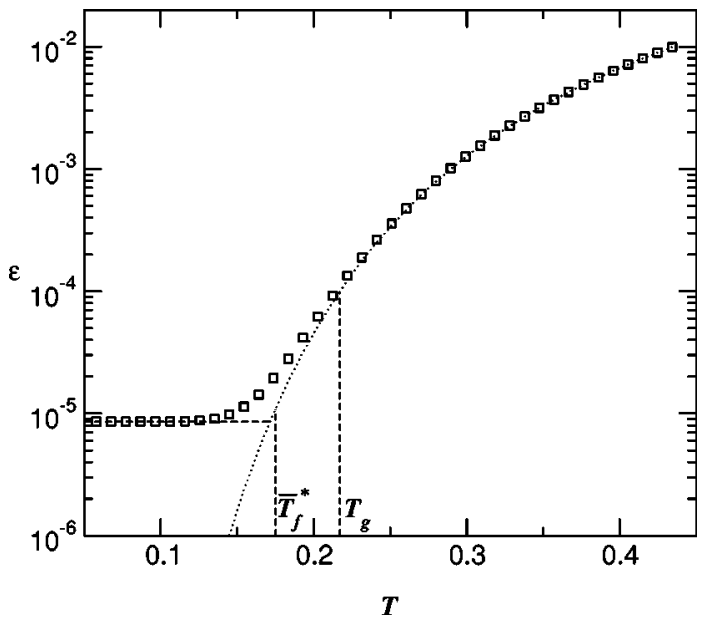

FIG. 5. Average dimensionless energy as a function of the dimensionless temperature $T$ in a cooling process (squares). The cooling law and the cooling rate are the same as in Fig. 4. The dotted line is the equilibrium energy, Eq. (2.6). The predicted value for the glass transition temperature $T_{g} \simeq 0.217$ is indicated. Also, the global fictive temperature $\bar{T}_{f}^{*}$, calculated from Eq. (5.41), is shown. It gives quite a good estimate of the temperature for which the equilibrium energy equals the residual energy.

$$
p_{\text {res }}(u) \simeq p_{e}\left[T_{f}^{*}(u)\right]=\left(r_{c} a u\right)^{1 / a u},
$$

which gives the same dependence of $p_{\text {res }}$ on $r_{c}$ as Eq. (5.19), except for a factor of the order of unity.

In Fig. 5 the dimensionless average energy $\varepsilon$, Eq. (5.20), is shown, for the same parameters as in Fig. 4. The predicted glass transition temperature $T_{g} \simeq 0.217$ is also indicated. It provides a good estimate for the beginning of the departure from the equilibrium curve. The residual value of the energy calculated from an asymptotic analysis of Eqs. (5.37) and (5.40) reads

$$
\varepsilon_{\mathrm{res}}=\int_{0}^{1} d u p_{\mathrm{res}}(u) \simeq \frac{\left(r_{c} a\right)^{1 / a}}{\left|\ln \left(r_{c} a\right)^{1 / a}\right|} .
$$

Comparison of Eqs. (5.41) and Eq. (5.22) also indicates a good agreement, the difference being again a factor of the order of unity. We can introduce a global fictive temperature $\bar{T}_{f}^{*}$ for the energy, as the temperature for which the equilibrium energy is the same as the energy of the system in the cooling process, when extrapolated to $T=0$, i.e.,

$$
\varepsilon_{\mathrm{res}}=\varepsilon_{e}\left(\bar{T}_{f}^{*}\right),
$$

This global fictive temperature $\bar{T}_{f}^{*}$ is related to the fictive temperatures of the levels $T_{f}^{*}(u)$ by

$$
\varepsilon_{e}\left(\bar{T}_{f}^{*}\right)=\int_{0}^{1} d u p_{e}\left[T_{f}^{*}(u)\right]
$$

The global fictive temperature is then a kind of average of the fictive temperatures $T_{f}^{*}(u)$, but the probability distribution of the frozen state is not the equilibrium distribution at the temperature $\bar{T}_{f}^{*}$. Each level freezes at its corresponding fictive temperature $T_{f}^{*}(u)$, and the probability of the frozen state $p_{\text {res }}(u)$ is approximately given by Eq. (5.40). For the values of the parameters considered in Fig. 5, Eq. (5.41) leads to $\bar{T}_{f}^{*} \simeq 0.175$, which is also a good approximation for the value of the temperature at which Eq. (5.42) holds. Finally, let us stress that similar results for the fictive temperatures can be derived by means of the qualitative reasonings developed in Ref. [28] and based on Hilbert's expansion.

\section{HEATING PROCESSES}

This section will be devoted to the study of heating processes after the system has been previously cooled down to very low temperatures. Then, the system is not initially at equilibrium, but the initial probability distribution $p\left(t_{0} ; u\right)$ corresponds to the final state reached in the cooling process. It is convenient to introduce a time $t_{h}<t_{0}$ such that $T\left(t_{h}\right)$ $=0$ if the heating law is extrapolated to times shorter than $t_{0}$. From Eq. (4.2) it follows that

$$
\begin{aligned}
p(t ; u)= & {\left[p\left(t_{0} ; u\right)-p_{e}\left(T_{0}\right)\right] \chi\left(t, t_{0} ; u\right)+p_{e}(T) } \\
& -\int_{t_{0}}^{t} d t^{\prime} \frac{d p_{e}\left(T^{\prime}\right)}{d T^{\prime}} \frac{d T^{\prime}}{d t^{\prime}} \chi\left(t, t^{\prime} ; u\right),
\end{aligned}
$$

where $\chi\left(t_{1}, t_{2} ; u\right)$ was defined in Eq. (5.6), and $T_{0}=T\left(t_{0}\right)$ is the initial temperature of the heating process. The first term on the RHS of Eq. (6.1) represents the decay of the initial nonequilibrium condition. For $T(t)$ in the high-temperature region it is $\chi\left(t, t_{0} ; u\right) \ll 1$, and this contribution can be neglected. This implies that $p(t ; u)$ reaches a behavior which is independent of the initial condition or, equivalently, independent of the previous cooling program. Moreover, the lower limit $t_{0}$ in the integral can be replaced by $t_{h}$, with a relative error that decreases as $t$ increases. Therefore, $p(t ; u)$ tends to the "normal"' solution

$$
p_{N}(T ; u)=p_{e}(T)-\int_{t_{h}}^{t} d t^{\prime} \frac{d p_{e}\left(T^{\prime}\right)}{d T^{\prime}} \frac{d T^{\prime}}{d t^{\prime}} \chi\left(t, t^{\prime} ; u\right) .
$$

Any arbitrary $p(t ; u)$ approaches $p_{N}(T ; u)$ for long enough times, corresponding to high temperatures. The existence of the normal solution for heating processes is not restricted to the hierarchical models considered in this paper, but it has been established for a quite general class of systems, whose dynamics is described in terms of a master equation [27]. In this context, the existence of the normal solution in our simplified, mean-field description of the hierarchical models, provides a consistency test of the approximations we have introduced starting from the master equation formulation. However, it cannot be assured that the special choice of the initial conditions leading to the normal solution in our meanfield-type approximation remains the same for a more exact analysis.

In the limit of high temperatures, $p_{N}(T ; u)$ must be closely related to Hilbert's expansion solution $p_{H}(T ; u)$, ana- 
lyzed in Sec. IV. In fact, since $p_{H}(T ; u)$ does not refer to any particular initial condition, it is to be expected that the normal solution coincides with Hilbert's expansion in their common range of validity. However, the normal solution is not restricted to near-equilibrium situations, and the system can approach the normal curve in a temperature range for which Hilbert's solution is not accurate. This point will be clearly illustrated below.

For the sake of simplicity, we will consider that the system is submitted to a cooling process given by Eq. (5.11), followed by a heating process of the form

$$
\frac{d p_{e}}{d t}=r_{h} p_{e}
$$

where $r_{h}>0$ is the heating rate. Using this heating law, we can express Eq. (6.1) in the simpler way:

$$
\begin{aligned}
p(t ; u)= & {\left[p_{0}(u)-p_{e 0}\right] \exp \left(-\frac{p_{e}^{a u}-p_{e 0}^{a u}}{r_{h} a u}\right)+p_{e} } \\
& -\int_{p_{e 0}}^{p_{e}} d p_{e}^{\prime} \exp \left(-\frac{p_{e}^{a u}-p_{e}^{\prime a u}}{r_{h} a u}\right) .
\end{aligned}
$$

Here we employ the same notation as in Eq. (5.12). Similarly,

$$
p_{N}(T ; u)=p_{e}-\int_{0}^{p_{e}} d p_{e}^{\prime} \exp \left(-\frac{p_{e}^{a u}-p_{e}^{\prime a u}}{r_{h} a u}\right) .
$$

The high-temperature regime for level $u$ is given by

$$
\frac{p_{e}^{a u}}{r_{h} a u} \gg 1
$$

and, in this limit, Eq. (6.5) reduces to

$$
p_{N}(T ; u) \simeq p_{e}-r_{h} p_{e}^{1-a u},
$$

which agrees with Hilbert's solution. Eq. (4.11), particularized for the heating law (6.3).

Figure 6 shows the evolution of $p(t ; u=1)$, as given by Eq. (6.4), in a heating process with $a=1$ and $r_{h}=10^{-4}$. Two different initial conditions have been considered, corresponding to slow previous coolings of the system with $r_{c}=10^{-4}$ and $r_{c}=10^{-6}$, respectively. In the figure, it is seen that both heating curves approach the normal solution, reaching it in a region where the normal curve represents a clear nonequilibrium state. Hilbert's expansion approximation, Eq. (6.7), is also plotted. It provides a good description for the linear correction around equilibrium of the normal curve, but fails for low enough temperatures. In fact, for the values of the parameters considered, Eq. (6.7) becomes negative for $T$ $<0.217$. Therefore, in general, Hilbert's solution cannot be used to estimate the normal curve over the whole range in which the system is well described by the normal solution, which depends on the details of the previous cooling process. In the figure, for the smallest cooling rate $r_{c}=10^{-6}$, the system reaches the normal curve for a temperature at which

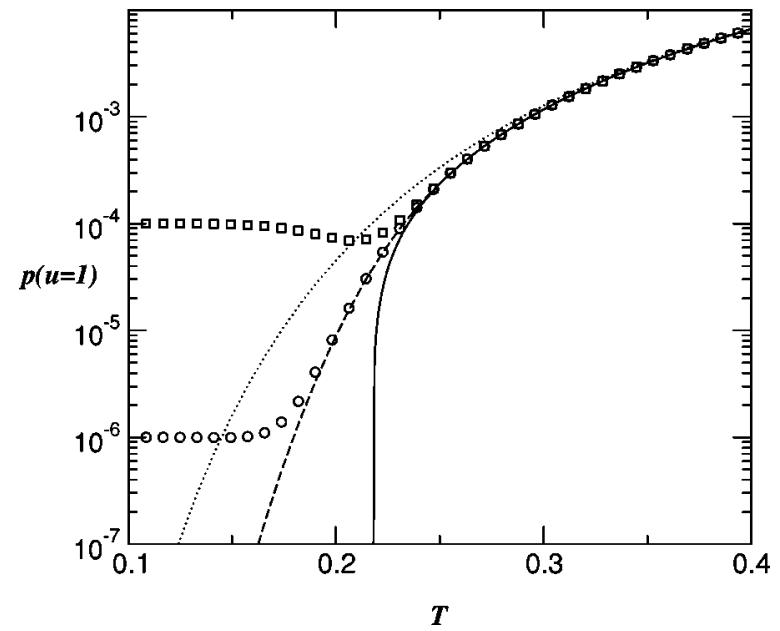

FIG. 6. Evolution of $p(u=1)$ in a heating process. The dotted line is the equilibrium curve. The heating law is Eq. (6.3), with a heating rate $r_{h}=10^{-4}$. The normal curve has been evaluated both by using Eq. (6.5) (dashed line) and Hilbert's expansion, Eq. (6.7) (solid line). Two different initial conditions for the heating process have been considered, both corresponding to previous coolings down to very low temperatures but with different rates, namely $r_{c}$ $=10^{-4}$ (squares) and $r_{c}=10^{-6}$ (circles). The cooling law was given by Eq. (5.11) in both cases.

Hilbert's expansion is not valid. This illustrates how the normal solution is relevant for far-from-equilibrium states, in which a linear theory in the deviations from equilibrium is not accurate.

In the experiments, either with supercooled liquids or with granular materials, the distribution function cannot be directly measured, but instead the average values of the relevant physical properties. In our model, we can consider the dimensionless energy $\varepsilon(t)$ [30], which is calculated by integrating $p(t ; u)$ over $u$. For the specific heating law we are considering, it follows from Eq. (6.4) that

$$
\begin{aligned}
\varepsilon(t)= & \int_{0}^{1} d u\left[p_{0}(u)-p_{e 0}\right] \exp \left(-\frac{p_{e}^{a u}-p_{e 0}^{a u}}{r_{h} a u}\right)+\varepsilon_{e}(T) \\
& -\int_{0}^{1} d u \int_{p_{e 0}}^{p_{e}} d p_{e}^{\prime} \exp \left(-\frac{p_{e}^{a u}-p_{e}^{\prime a u}}{r_{h} a u}\right) .
\end{aligned}
$$

There is also a normal curve for the energy $\varepsilon(T)$, which can be obtained by integration of the normal probability distribution, Eq. (6.5), i.e.,

$$
\varepsilon_{N}(t)=\varepsilon_{e}(T)-\int_{0}^{1} d u \int_{0}^{p_{e}} d p_{e}^{\prime} \exp \left(-\frac{p_{e}^{a u}-p_{e}^{\prime a u}}{r_{h} a u}\right) .
$$

For long enough times, independently of the initial probability distribution $p_{0}(u), \varepsilon(t)$ will approach $\varepsilon_{N}(T)$, as a consequence of the tendency of $p(t ; u)$ towards $p_{N}(T ; u)$. The time regime in which $\varepsilon(t)$ practically agrees with $\varepsilon_{N}(T)$ corresponds to the condition given by Eq. (6.6) being verified for all $u$, i.e., when it is fulfilled by the slowest modes, $u=1$. Therefore, the approach to the normal curve is con- 


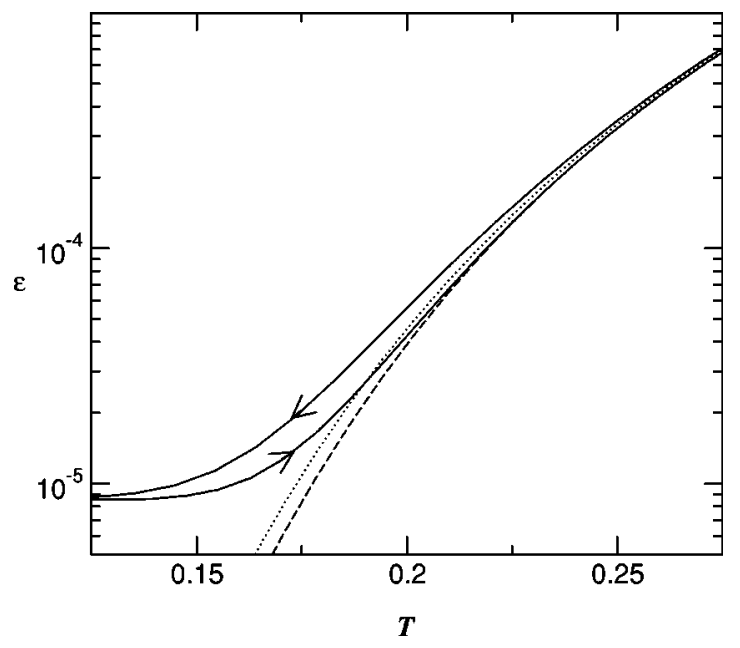

FIG. 7. Hysteresis of the dimensionless energy $\varepsilon$ in a thermal cycle. As in the previous figures, the cooling law is Eq. (5.11) and the heating law is Eq. (6.3). The cooling and heating rates are the same, $r_{c}=r_{h}=10^{-4}$. The dotted line is the equilibrium energy, and the dashed line is the normal curve for the heating process. The arrows over the solid lines indicate the variation of the temperature in each process. In the heating process, the system approaches the normal solution, crossing the equilibrium curve.

trolled by the slowest level $u=1$. For very high temperatures, Hilbert's result (4.13) holds and, particularizing for the heating law of Eq. (6.3), we get

$$
\varepsilon_{N}(T) \simeq \varepsilon_{H}(T)=p_{e}-r_{h} p_{e} \frac{p_{e}^{-a}-1}{a\left|\ln p_{e}\right|}
$$

An important feature of the normal curve is that

$$
\varepsilon_{N}(T) \leqslant \varepsilon_{e}(T),
$$

for all temperatures. This property, together with the inequality, Eq. (5.4), for cooling processes, explains the hysteresis effects exhibited by the model when submitted to a thermal cycle. These effects are similar to the experimentally observed behavior in glass-forming liquids [22] and other complex systems, such as granular materials [26]. In Fig. 7 a hysteresis cycle of the energy is shown for $r_{c}=r_{h}=10^{-4}$. In the cooling process the energy is greater than the corresponding equilibrium value, while in the heating process the tendency of the system to approach the normal curve, which verifies Eq. (6.11), makes the system overtake the equilibrium curve. Only for very high temperatures, where Hilbert's expansion is accurate, does the normal solution tend to the equilibrium one from below. Comparison of Figs. 6 and 7 shows that the separation of the normal solution for the energy from the equilibrium curve is smaller than that of the slowest level probability $p(u=1)$. This is due to the contribution of the other modes, which reach their equilibrium values for lower temperatures.

In our opinion, the explanation of the hysteretic behavior observed in real systems must be similar to the above dis- cussion. This idea is supported by the fact that in other models based on a master equation formalism, analogous results have been found $[28,31,35]$. Also, Hilbert's expansion, although of limited validity, leads to the conclusion that cooling and heating curves are at opposite sides of the equilibrium curve.

\section{DISCUSSION}

In this paper, we have analyzed a simple spin model with hierarchically constrained dynamics. The spins are classified into levels, and a spin in level $n+1$ is able to relax only if a certain number of spins in level $n$ are in the up (excited) state. Starting from a master equation formulation of the dynamics, a mean-field approximation was introduced. The result is a generalization of the model of Palmer et al. [7], with a closed equation for the evolution of the probability of finding a given spin in the up state. Each level has a characteristic relaxation rate, which is a function of the temperature of the system. Two sets of parameters characterize the model: the number of spins in level $n, N_{n}$, and the number $\mu_{n}$ of spins in level $n$ involved in the facilitation of the relaxation of a spin in level $n+1$. We have chosen the simplest possibility, namely, that $\mu_{n}$ and $N_{n}$ are proportional to each other, which leads to a relaxation behavior that is independent of the level populations $N_{n}$. In relaxation processes at constant temperature, the system displays linear logarithmic decay [11]. This is a characteristic feature of the behavior of a wide variety of complex systems, including structural glasses, spin glasses, protein models, and powders.

A key point in our approach to the problem of hierarchically constrained dynamics is the introduction of the "meanfield" approximation, which allows us to reduce the initial, rather involved, problem to a solvable one. This approximation is based on the physical idea that hierarchical constraints render the characteristic time scales of the different levels clearly separated. This is indeed the case in the lowtemperature region, since the ratio $\tau_{n} / \tau_{n-1}$ of the characteristic times of levels $n$ and $n-1$, as given by Eq. (3.5), diverges for $T \rightarrow 0$.

The model has also been used to study processes in which the temperature changes in time in an arbitrary way. The general solution for the time-dependent distribution function can be explicitly written, and the average energy has a form resembling that of Narayanaswami's phenomenological theory of glass-forming liquids [25]. By means of Hilbert's expansion we have constructed an approximate solution for the probability distribution. This expression is valid in the limit of very high temperatures and for situations where the system is in the linear around equilibrium region. The behavior of Hilbert's solution is formally identical to the one previously found for a very general class of systems described by master equations [28], despite the mean-field character of the simplified model considered here. Hilbert's expansion predicts the existence of hysteresis effects when the system is first cooled down to low temperatures and, afterwards, reheated to high temperatures. This is because the average energy is at opposite sides of the equilibrium curve for cooling and heating processes. 
Another point we have addressed is the evolution of the system in continuous cooling processes. A phenomenon similar to the laboratory glass transition shows up, and the system departs from equilibrium at low temperatures. The magnitude of this separation can be measured by the values of the residual properties, which have been analytically computed. A simple but physically appealing argument is presented, in order to understand the origin of this glassy behavior. Each level in the system becomes frozen at the equilibrium value corresponding to a temperature, called the fictive temperature of the level, such that the average number of transitions per spin in that level until reaching $T=0$, following the prescribed cooling program, equals unity. A similar argument has been previously used in other models $[28,31,35]$. Here, an analytical derivation is presented. This provides a theoretical basis for the concept of fictive temperature and clarifies the accuracy of the results following from the qualitative argument. We have compared them with the values of the residual properties obtained numerically as well as with those following from asymptotic analysis calculations.

Finally, heating processes have also been analyzed. More specifically, we have considered heating processes following a continuous cooling of the system down to very low temperatures. In the description of heating processes, the socalled normal curve plays a fundamental role. The hysteresis effects observed when the system is first cooled and afterwards reheated appear because of the trend of the system to approach the normal solution, along the heating evolution.
This is analogous to previous results found in models for structural glasses [31,35] and for granular systems [28]. Thus, we think it would be worth investigating whether a similar curve does exist for real complex systems showing this kind of hysteretic behavior.

Although a particular simple model with hierarchically constrained dynamics has been considered in this paper, most of the physical ideas developed seem very general. The validity of Hilbert's expansion for high temperatures, the natural appearance of the concept of fictive temperature, leading a physically appealing description of the laboratory glass transition, the existence of the normal solution, and its fundamental role in explaining the hysteresis effects are results that are not restricted to the present model. In fact, similar results appear in a quite general class of systems described by master equations $[28,31,35]$. Of course, the details of the dynamical behavior depend on the specific model we are dealing with. On the other hand, the general picture developed here, where the heating and cooling experiments in glasses appear as purely kinetic and relatively simple to understand, might not be valid for real and much more complex systems.

\section{ACKNOWLEDGMENT}

This research has been partially supported by the Dirección General de Investigación Científica y Técnica (Spain) through Grant No. PB98-1124.
[1] G. H. Fredrickson and H. C. Andersen, Phys. Rev. Lett. 53, 1244 (1984).

[2] G. H. Fredrickson and S. A. Brawer, J. Chem. Phys. 84, 3351 (1986).

[3] S. Eisinger and J. Jäckle, J. Stat. Phys. 73, 643 (1993).

[4] P. Harrowell, Phys. Rev. E 48, 4359 (1993).

[5] F. Mauch and J. Jäckle, Physica A 262, 98 (1999).

[6] J. J. Brey, A. Prados, and B. Sánchez-Rey, Phys. Rev. E 60, 5685 (1999).

[7] R. G. Palmer, D. L. Stein, E. Abrahams, and P. W. Anderson, Phys. Rev. Lett. 53, 958 (1984).

[8] M. Skorobogatiy, H. Guo, and M. Zuckermann, J. Chem. Phys. 109, 2528 (1998).

[9] V. V. Bredikhin, V. A. Sidorov, O. B. Tsiok, E. V. Gurovich, and L. G. Khvostantsev, Europhys. Lett. 18, 111 (1992).

[10] O. B. Tsiok, V. A. Sidorov, V. V. Bredikhin, L. G. Khvostantsev, V. N. Troitskiy, and L. I. Trusov, Phys. Rev. B 51, 12127 (1995).

[11] J. J. Brey and A. Prados, Phys. Rev. E 63, 021108 (2001).

[12] P. Nordblad, P. Svedlindh, L. Lundgren, and L. Sandlund, Phys. Rev. B 33, 645 (1986).

[13] E. I. Shakhnovich and A. M. Gutin, Europhys. Lett. 9, 569 (1989).

[14] H. M. Jaeger, C-h. Liu, and S. R. Nagel, Phys. Rev. Lett. 62, 40 (1989).

[15] A. Mehta, R. J. Needs, and S. Dattagupta, J. Stat. Phys. 68, 1131 (1992).
[16] O. B. Tsiok, V. Brazhkin, A. G. Lyapin, and L. G. Khovstantsev, Phys. Rev. Lett. 80, 999 (1998).

[17] L. Fabbian, W. Götze, F. Sciotino, P. Tartaglia, and F. Thiery, Phys. Rev. E 59, R1347 (1999).

[18] V. G. Karpov and M. Grimsditch, Phys. Rev. B 48, 6941 (1993).

[19] A. Fernández and G. Appignanesi, Phys. Rev. Lett. 78, 2668 (1997).

[20] S. F. Edwards and R. B. S. Oakesshott, Physica A 157, 1080 (1989).

[21] A. Mehta and S. F. Edwards, Physica A 157, 1091 (1989).

[22] G. W. Scherer, Relaxation in Glass and Composites (Wiley, New York, 1986).

[23] S. A. Brawer, Relaxation in Viscous Liquids and Glasses (American Ceramic Society, Columbus, OH, 1985).

[24] G. W. Scherer, J. Non-Cryst. Solids 123, 75 (1990).

[25] O. S. Narayanaswami, J. Am. Ceram. Soc. 54, 471 (1971).

[26] E. R. Nowak, J. B. Knight, E. Ben-Naim, H. M. Jaeger, and S. R. Nagel, Phys. Rev. E 57, 1971 (1998).

[27] J. J. Brey and A. Prados, Phys. Rev. E 47, 1541 (1993).

[28] A. Prados, J. J. Brey, and B. Sánchez-Rey, Physica A 284, 277 (2000).

[29] R. J. Glauber, J. Math. Phys. 4, 294 (1963).

[30] In a granular system, $\varepsilon$ represents the average volume of the system and $T$ is Edward's compactivity $X$.

[31] J. J. Brey and A. Prados, Phys. Rev. B 49, 984 (1994). 
[32] S. A. Langer, A. T. Dorsey, and J. P. Sethna, Phys. Rev. B 40, 345 (1989).

[33] S. J. Cornell, K. Kaski, and R. B. Stinchcombe, Phys. Rev. B 44, 12263 (1991).
[34] J. J. Brey and A. Prados, Phys. Rev. B 43, 8350 (1991).

[35] A. Prados, J. J. Brey, and B. Sánchez-Rey, Phys. Rev. B 55, 6343 (1997).

[36] J. C. Dyre, Phys. Rev. Lett. 58, 792 (1987). 